\title{
Angiolipoma intracraniano adjacente a aneurisma sacular cerebral: relato de caso e revisão da literatura
}

\section{Intracranial Angiolipoma Associated with Cerebral Saccular Aneurysm: Case Report and Review of Literature}

Robinson A. M. Marques ${ }^{1}$ Alexandre Novicki Francisco ${ }^{2}$ Luiz Marcio de Brito Marinho Segundo ${ }^{3}$ Marcos V. C. Maldaun ${ }^{4}$

${ }^{1}$ Neurocirurgião do Hospital Universitário Cajuru, Curitiba,

Address for correspondence Robinson Marques, MD, Serviço de Paraná, Brasil Neurocirurgia, Hospital Universitário Cajuru, Av. São José, 300, Cristo

${ }^{2}$ Chefe de Serviço, Hospital Universitário Cajuru, Curitiba, Rei, Curitiba, PR, Brasil, CEP 80050-350

Paraná, Brasil (e-mail: menegottom@yahoo.com.br).

${ }^{3}$ Médico-Residente de Neurocirurgia do Hospital Universitário Cajuru, Curitiba, Paraná, Brasil

${ }^{4}$ Coordenador do Núcleo de Neurologia e Neurocirurgia, Hospital

Sírio Libanês, São Paulo, Brasil

Arq Bras Neurocir 2015;34:148-152.

\section{Resumo \\ Palavras-Chave \\ - angiolipoma intracraniano \\ - aneurisma sacular \\ - cefaleia \\ - neoplasia cerebral}

Angiolipomas são neoplasias benignas compostas por adipócitos maduros e proliferação vascular angiomatosa de rara ocorrência no Sistema Nervoso Central. É relatado o caso de angiolipoma em cisterna silviana adjacente a aneurisma sacular de artéria cerebral média. São discutidas as características imagenológicas do tumor e a possibilidade de uma origem comum com lesões vasculares intracranianas. Este é o primeiro caso relatado de angiolipoma intracraniano associado a aneurisma de artéria cerebral média.

Angiolipomas are benign neoplasms composed of mature fat cells and vascular angiomatous proliferation of rare incidence in Central Nervous System.It's related a case of angiolipoma in Sylvian fissure associated with cerebral saccular aneurysm of medial cerebral artery. Imagenologic characteristics of the tumor and a possible common origin with intracranial vascular lesions are discussed. This is the first reported case of intracranial angiolipoma involving medial cerebral artery aneurysm.

\section{Introdução}

Lipomas são tumores benignos do tecido adiposo, sendo o mais comum dentre os tumores de partes moles na vida adulta. O subtipo mais frequente é o lipoma convencional, que se caracteriza por uma massa bem encapsulada de adipócitos de tamanhos variados, localizando-se principalmente nas extremidades proximais e tronco. Os received

May 13, 2014

accepted

April 31, 2015
DOI http://dx.doi.org/ 10.1055/s-0035-1554051. ISSN 0103-5355.
Copyright $\odot 2015$ by Thieme Publicações License terms Ltda, Rio de Janeiro, Brazil $(\circledast) \Theta \Theta$ 
angiolipomas (AGL), outro subtipo de lipoma, são neoplasias benignas que representam 5 a $17 \%$ de todos os lipomas e acometem principalmente adolescentes e adultos jovens. A associação de tecido vascular com tecido adiposo já havia sido relatada por Bowen em 1912, mas a histologia dos AGL só foi descrita em 1960 por Howard e Helwing. ${ }^{1}$

Os AGL intracranianos são raros, sendo a região da base do crânio o local mais comumente afetado, em especial a região parasselar. ${ }^{2}$ Os AGL intracranianos associados a aneurismas cerebrais são ainda menos frequentes, tendo sido descritos poucos casos na literatura ${ }^{3,4}$, por isso a importância do relato de mais um caso raro como este, no qual foi diagnosticado um AGL adjacente a um aneurisma sacular em artéria cerebral média (ACM).

\section{Materiais e Métodos}

Estudo retrospectivo com análise de prontuário, observação clínica do paciente e interpretação de exames complementares, associado à revisão da literatura com busca nos bancos de dados do PubMed, Lilacs, Scielo, Embase e Journal of Neurosurgery com os seguintes termos isolados ou combinados: angiolipoma intracraniano, angiolipoma intracraneano, intracranial angiolipoma, lipoma intracranian, lipoma intracraneano, intracranial lipoma, aneurisma cerebral, intracranial aneurysm, sem restrição temporal. Foram selecionados os artigos com temas relacionados ao caso descrito.

\section{Relato do Caso}

Paciente masculino, 35 anos, admitido em nosso serviço devido a quadro de cefaléia holocraniana de forte intensidade, persistente e refratária à medicação analgésica, com 4 dias de evolução.

História prévia de clipagem de aneurisma em ACM esquerda em outro serviço 6 meses antes, quando os exames de imagem realizados evidenciaram: (1) à tomografia computadorizada (TC) de crânio, pequena imagem ovalada, hipo- densa (-77 unidades Hounsfield), medindo cerca de $14 \times 8 \mathrm{~mm}$, localizada na profundidade da fissura silviana esquerda, sugerindo o diagnóstico de lipoma; (2) à ressonância magnética (RM) de encéfalo, pequena lesão hiperintensa nas sequências ponderadas em T1 e T2 e hipointensa nas sequências com supressão de gordura, além de pequena área de sinal flow void na sequência ponderada em T2, também na fissura silviana esquerda, sugestiva de lesão aneurismática; (3) à angiorressonância cerebral, presença de um aneurisma sacular, parcialmente trombosado, orientado para cima e para frente, medindo $5 \mathrm{~mm}$ em seu maior diâmetro, na bifurcação da ACM esquerda (-Figs. 1A, 1C e 2).

0 exame físico à admissão apresentou-se dentro da normalidade. Exame de TC de crânio evidenciou lesão hipodensa, bem delimitada, com aspecto sugestivo de tecido gorduroso na topografia da fissura silviana esquerda adjacente a artefato metálico (clipe de aneurisma). Punção lombar com líquor normal. Exame de arteriografia cerebral diagnóstica evidenciou aneurisma sacular medindo aproximadamente $3 \mathrm{~mm}$ em seu maior diâmetro, na bifurcação da ACM esquerda (-Figs. 3A e $\mathbf{3 B}$ ).

$\mathrm{O}$ paciente foi submetido à cirurgia para clipagem do aneurisma através de craniotomia pterional esquerda. Durante o procedimento cirúrgico foi observado o clipe de aneurisma do procedimento prévio envolto por massa tumoral de cor róseo-amarelada entremeada pelos ramos da artéria cerebral média esquerda na transição M1-M2. Para a identificação dos ramos da artéria e do aneurisma foi necessária a ressecção da massa tumoral, realizada com técnica microcirúrgica, que se demonstrou extremamente difícil devido a aderência do tumor aos vasos. A ressecção foi interrompida após a exposição suficiente para a clipagem segura do aneurisma (-Figs. 4A e 4B). A cirurgia transcorreu sem intercorrências, e o paciente apresentou evolução pósoperatória favorável, recebendo alta hospitalar no quinto dia pós-operatório.

O seguimento do paciente se deu através de acompanhamento ambulatorial e exames de tomografia e angiografia, que não apresentaram complicações tardias nem
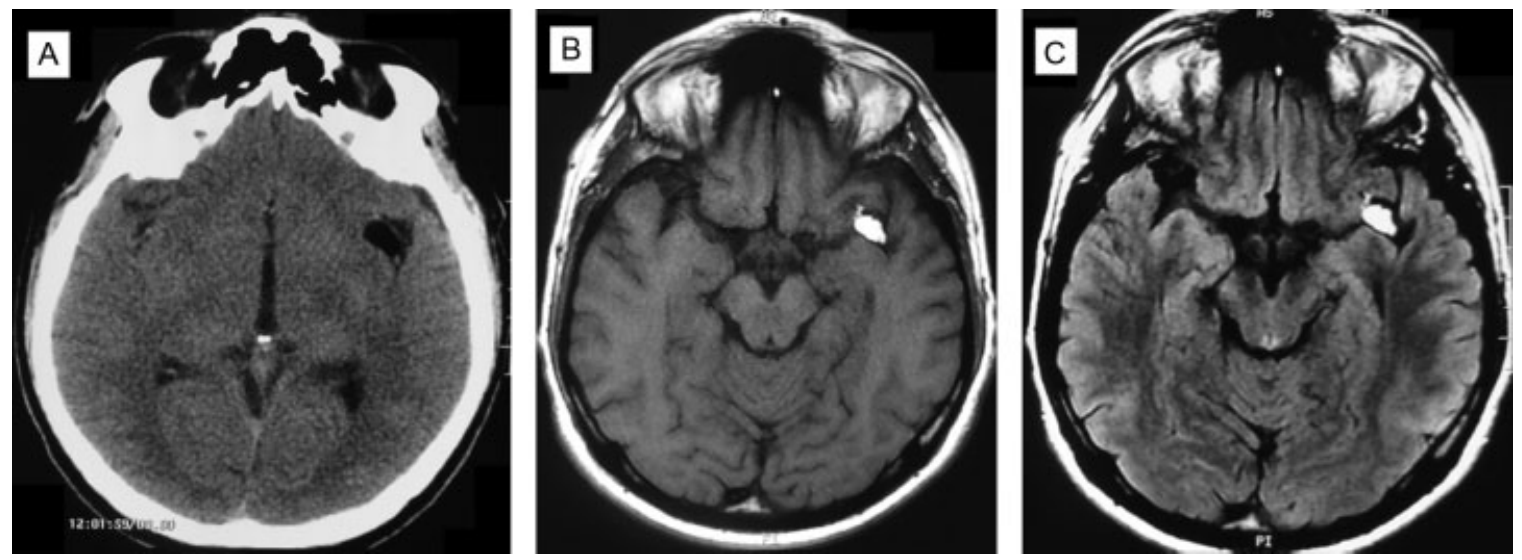

Fig. 1 Exames de imagem. (A) Tomografia axial de crânio. (B) Ressonância magnética ponderada em T1. (C) Ressonância magnética FLAIR em T2. 


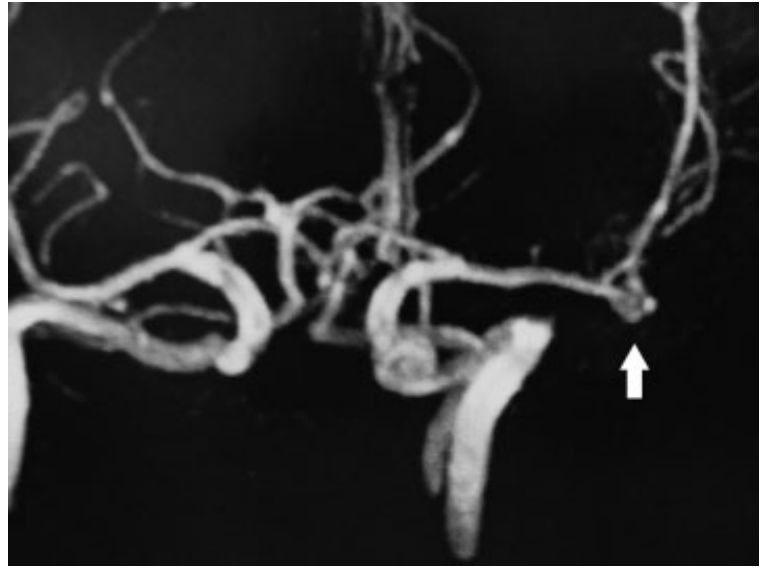

Fig. 2 Angiorressonância encefálica. Seta branca: aneurisma da artéria cerebral média esquerda.

recidiva tumoral ou aneurisma intracraniano. Arteriografia cerebral diagnóstica de controle realizada no terceiro mês pós-operatório não evidenciou outras alterações na vascularização encefálica e inclusive comprovou a oclusão total
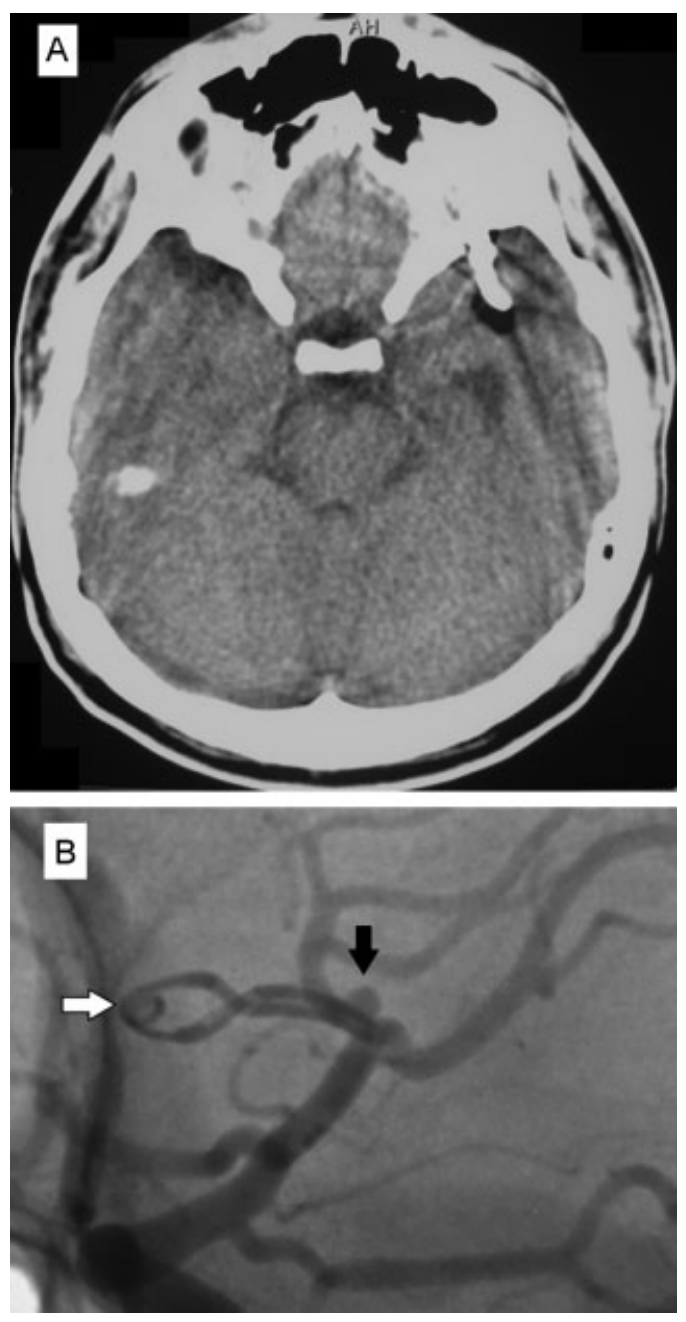

Fig. 3 (A) Tomografia axial de crânio. (B) Angiografia. Seta branca: clipe de aneurisma. Seta preta: aneurisma da artéria cerebral média esquerda. do aneurisma cerebral recém-operado. O laudo do estudo anatomopatológico da lesão ressecada durante a cirurgia comprovou a presença de tecido adiposo maduro sem atipias e com vasos de permeio, compatível com o diagnóstico de AGL (-Fig. 5 ).

\section{Discussão}

Os angiolipomas (AGL), descritos inicialmente por Bowen em 1912, caracterizados como um subtipo de lipoma, foram aceitos como entidade clínica após o trabalho de Howard e Helwig em 1960. ${ }^{1}$ São compostos por lipócitos maduros e proliferação vascular angiomatosa em seu interior. Segundo Lin e Lin, ${ }^{1}$ os AGL são subdivididos em infiltrativos e não infiltrativos. Os critérios de inclusão utilizados neste trabalho para o diagnóstico dos angiolipomas foram:

- formação tumoral com ou sem cápsula;

- evidência microscópica de lipócitos maduros perfazendo ao mínimo $50 \%$ da massa tumoral;

- evidência microscópica de proliferação angiomatosa no interior do tumor.

A incidência de AGL na série de Howard e Helwig foi de $17 \%$ de todos os lipomas, $5 \%$ na série de Lin e Lin e $47,3 \%$ na série de Ochoa Ryes et al. ${ }^{1,5}$

Geralmente os AGL se apresentam como tumores subcutâneos múltiplos, dolorosos, em adultos jovens. As extremidades inferiores são as áreas mais acometidas em 48\% dos casos, seguidas do pescoço e ombros. Os locais menos frequentes compreendem o espaço epidural espinhal, principalmente na região torácica e tecido retroperitoneal. ${ }^{1,6-16}$

Angiolipomas intracranianos são raros. A partir do relato de Takeuchiu et al. em 1981, poucos casos foram relatados na literatura. Em oposição aos lipomas intracranianos que ocorrem preferencialmente na linha média, os AGL intracranianos descritos localizavam-se na base do crânio, em região parasselar em íntimo contato com o seio cavernoso. 2,4,17-20 Outros sítios descritos incluem o tálamo, ${ }^{21}$, intraventricu$\operatorname{lar}^{22}$ e mastóideo. ${ }^{23}$

Existem relatos de lipomas em fissura silviana ${ }^{24,25}$ e de um caso de lipoma em fissura silviana associado a aneurisma de artéria cerebral média. ${ }^{26}$ Nosso estudo apresenta o primeiro caso de AGL em fissura silviana associado a aneurisma da artéria cerebral média.

As manifestações clínicas dos AGL intracranianos geralmente estão relacionadas ao efeito compressivo sobre estruturas vizinhas; em alguns casos relatados os AGL eram assintomáticos sendo encontrados como achado de exame. ${ }^{2,4,17,20} \mathrm{Em}$ um destes casos, a manifestação inicial do AGL foi hemorragia subaracnóidea. ${ }^{27}$

A origem dos AGL é incerta. Alguns autores acreditam que estão relacionados a defeitos da crista neural. ${ }^{3}$ Schievink et al. descrevem dois casos de angiolipomatose e dissecção arterial cervical espontânea e concluem que defeitos da crista neural podem provocar angiolipomatose e malformação da túnica média das artérias do pescoço e cabeça, o que poderia levar a formação de aneurismas e dissecções arteriais. $^{3}$ 

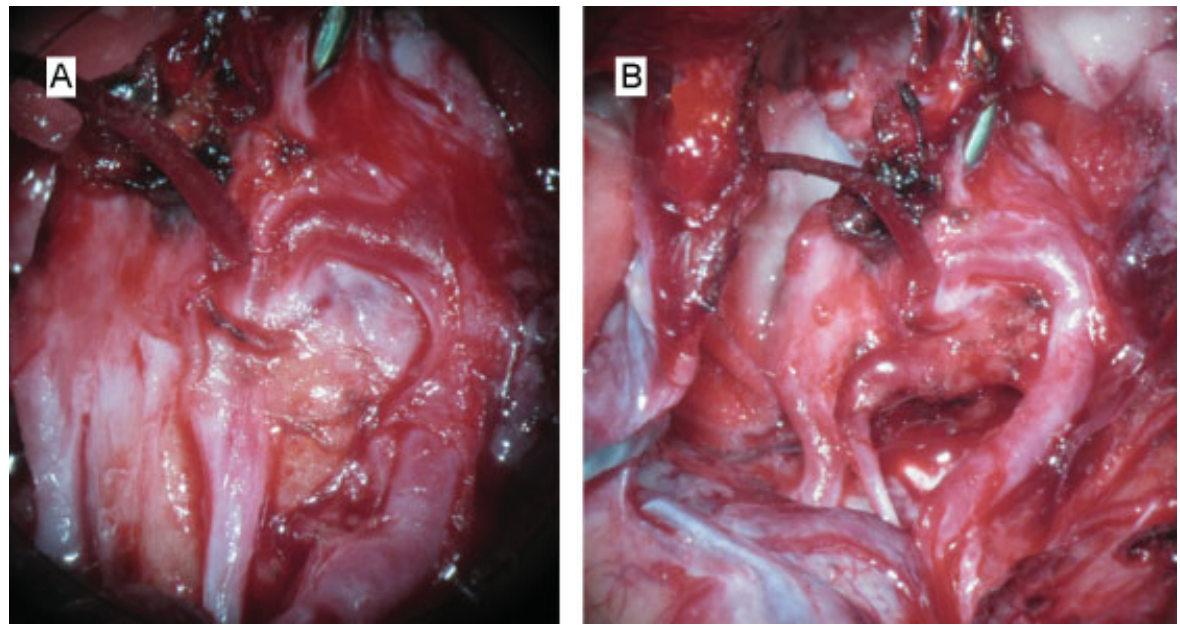

Fig. 4 (A) Angiolipoma envolvendo ramos da artéria cerebral média esquerda. (B) Ressecção parcial do angiolipoma e clipagem do aneurisma da artéria cerebral média.

Stevenson et al. descrevem um caso de múltiplos aneurismas cerebrais associados a múltiplos meningiomas e angiolipomas subcutâneos sugerindo uma origem genética comum. ${ }^{28}$ Nos AGL localizados em região parasselar, François et al. teorizam que sua origem se deve a células adiposas, remanescentes fetais, localizadas no espaço interperiosteal dural, entre os folhetos do seio cavernoso. ${ }^{17}$ Futami et al. sugerem que lipomas intracranianos localizados em fissura silviana tem origem malformativa advinda da persistência e diferenciação anormal da meninge primitiva e que os aneurismas associados poderiam dever-se a deficiência estrutural congênita que ocorreria ao mesmo tempo que a formação dos lipomas. ${ }^{26}$

O diagnóstico dos AGL intracranianos é feito através de exames de imagem. À TC esses tumores podem apresentar-se

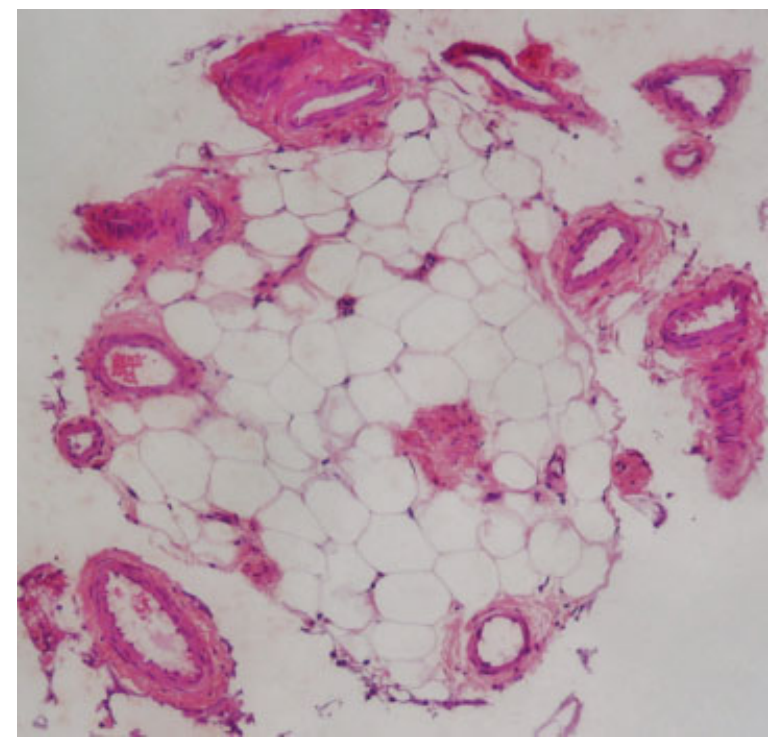

Fig. 5 Imagem de lâmina demonstrando tecido adiposo maduro e estrutura vascular angiomatosa, sem atipias, condizente com o diagnóstico de angiolipoma (HE 100x). como lesões hipodensas, homogêneas ou heterogêneas de acordo com a relação de tecido gorduroso e vascular em seu interior, com captação do meio de contraste. À RM os AGL apresentam-se hiperintensos nas sequências T1 e T2, mas aparecem hipointensos nas sequências com supressão de gordura. Geralmente há forte captação do meio de contraste. Estas características podem auxiliar no diagnóstico diferencial de outras neoplasias cerebrais como lipomas, cistos dermoides, epidermoides e teratomas, ${ }^{2,4,20,29}$ sendo a RM o exame de escolha para o diagnóstico dos AGL intracranianos.

Alguns casos de AGL intracranianos são tratados conservadoramente, principalmente nos pacientes assintomáticos, em virtude do caráter benigno destes tumores, mas a ressecção cirúrgica dos mesmos se faz necessária quando sintomas graves ou refratários estão associados. Devido a seu componente vascular, pode ocorrer sangramento profuso durante sua ressecção impedindo a remoção completa. ${ }^{2,4,20}$ Quando localizados em fissura silviana, tomando como base os lipomas nesta situação, ${ }^{25}$ sua remoção completa pode ser perigosa devido a sua íntima relação com ramos da ACM.

\section{Conclusão}

Os angiolipomas intracranianos são raros. O local mais frequente de acometimento intracraniano é a região parasselar em íntimo contato com o seio cavernoso. A ressecção cirúrgica desses tumores pode estar associada a sangramento profuso impedindo a remoção completa. Este é o primeiro caso relatado de AGL em fissura silviana associado a aneurisma de ACM.

\section{Referências}

1 Lin JJ, Lin F. Two entities in angiolipoma. A study of 459 cases of lipoma with review of literature on infiltrating angiolipoma. Cancer 1974;34(3):720-727 
2 Pirotte B, Krischek B, Levivier M, Bolyn S, Brucher JM, Brotchi J. Diagnostic and microsurgical presentation of intracranial angiolipomas. Case report and review of the literature. J Neurosurg 1998;88(1):129-132

3 Schievink WI, Thompson RC, Yong WH. A syndrome of spontaneous cerebral and cervical artery dissections with angiolipomatosis. Report of two cases. J Neurosurg 2003;98(5):1124-1127

4 Takeuchi J, Handa H, Keyaki A, Haibara H, Ozaki S. Intracranial angiolipoma. Surg Neurol 1981;15(2):110-113

5 Ochoa Reyes J, Peniche J, Arellano Mendoza I, Mercadillo P. Lipoma y angiolipoma: estúdio clínico patológico, comparativo y retrospectivo em 260 pacientes. Dermatol Rev Mex 1994;38(6):393-397

6 Aguiar PH, Plese JP, Rosemberg S, et al. Angiolipoma de coluna torácica: relato de caso. Arq Bras Neurocir 1996;15(2):103-107

7 Akhaddar A, Albouzidi A, Elmostarchid B, Gazzaz M, Boucetta M. Sudden onset of paraplegia caused by hemorrhagic spinal epidural angiolipoma. A case report. Eur Spine J 2008;17(Suppl 2):S296-S298

8 Balbo RJ, Araújo JFM, Melro CAM, Iafigliola MG, Valvassore FR. Angiolipoma epidural torácico. Relato de caso. Arq Neuropsiquiatr 1995;53(3-B):659-661

9 Blount JP, Elton S. Spinal lipomas. Neurosurg Focus 2001;10(1):e3

10 Gelabert-González M, García-Allut A. Spinal extradural angiolipoma: report of two cases and review of the literature. Eur Spine J 2009;18(3):324-335

11 Machado Filho JA, Fabião Neto OM, Ordovás CA, Hack I, Gallo P. Angiolipoma intrarraquidiano: relato de caso. Arq Bras Neurocir 1993;12(2):141-144

12 Palmieri GN, Borré GE, Borré DG. Angiolipoma epidural: presentación de caso y revisión de literatura. Rev Argent Radiol 2000; 64(3):231-234

13 Park JH, Jeon SR, Rhim SC, Roh SW. Lumbar spinal extradural angiolipoma: case report and review of the literature. J Korean Neurosurg Soc 2008;44(4):265-267

14 do Souto AA, Domingues FS, Chimelli L, Lemos AM. Lumbosacral angiolipoma: case report. Arq Neuropsiquiatr 2003;61 (2A):269-273

15 Trewhella RN, Gutiérrez JP, Cubillos AL, Rjas GH, Olivares O, Ramis C. Angiolipoma espinal diagnosticado durante el embarazo. Rev Chil Obstet Ginecol 2003;68(3):245-247
16 Turgut M. [Lumbosacral angiolipoma]. Arq Neuropsiquiatr 2004; 62(1):185-186, author reply 186

17 François P, Zemmoura I, Fouquet AMB, Jan M, Velut S. Lateral sellar angiolipoma: a tumor illustrative of the extradural compartment of the neural axis. J Neurosurg 2010;113(5): 1053-1058

18 Gerber SS, Plotkin R. Lipoma of the corpus callosum. Case report. J Neurosurg 1982;57(2):281-285

19 Loddenkemper T, Morris HH III, Diehl B, Lachhwani DK. Intracranial lipomas and epilepsy. J Neurol 2006;253(5): 590-593

20 Wilkins PR, Hoddinott C, Hourihan MD, Davies KG, Sebugwawo S, Weeks RD. Intracranial angiolipoma. J Neurol Neurosurg Psychiatry 1987;50(8):1057-1059

21 Shuangshoti S, Vajragupta L. Angiolipoma of thalamus presenting with abrupt onset suggestive of cerebrovascular disease. Clin Neuropathol 1995;14(2):82-85

22 Lee HK, Ghani AR, Sayuthi S, et al. Intraventricular angiolipoma. Med J Malaysia 2007;62(4):339-340

23 Viada Lozano J, Moyano SL, Sepúlveda SJC. Angiolipoma mastoideo. Rev Otorrinolaringol Cir Cabeza Cuello 1998;58(1):31-34

24 Feldman RP, Marcovici A, LaSala PA. Intracranial lipoma of the sylvian fissure. Case report and review of the literature. J Neurosurg 2001;94(3):515-519

25 Hatashita S, Sakakibara T, Ishii S. Lipoma of the insula. Case report. J Neurosurg 1983;58(2):300-302

26 Futami K, Kimura A, Yamashita J. Intracranial lipoma associated with cerebral saccular aneurysm. Case report. J Neurosurg 1992; 77(4):640-642

27 Vilela P, Saraiva P, Goulão A. Intracranial angiolipoma as cause of subarachnoid haemorrhage. Case report and review of the literature. Neuroradiology 2005;47(2):91-96

28 Stevenson JC, Choksey MS, McMahon J, Crawford PJ. Multiple cerebral aneurysms, multiple meningiomas and multiple subcutaneous angiolipomas: a case report. Br J Neurosurg 1994;8(4): 477-481

29 Friedman RB, Segal R, Latchaw RE. Computerized tomographic and magnetic resonance imaging of intracranial lipoma. Case report. J Neurosurg 1986;65(3):407-410 